\title{
CRESCIMENTO E NUTRIÇÃO MINERAL DE MUDAS DE BARBATIMÃO SOB EFEITO DA OMISSÃO DE NUTRIENTES
}

\author{
Leandro Carlos $^{1}$, Nelson Venturin², Renato Luiz Grisi Macedo ${ }^{2}$, Emilio Manabu Higashikawa ${ }^{1}$ \\ ${ }^{1}$ Eng. Florestal, M.Sc., Depto. de Ciências Florestais, UFLA, Lavras MG, Brasil- 1cmaestro@gmail.com; \\ emilio.higashikawa@gmail.com \\ 2Eng. Florestal, Dr., Depto. de Ciências Florestais, UFLA, Lavras MG, Brasil - venturin@dcf.ufla.br; rlgrisi@ufla.br
}

Recebido para publicação: 08/10/2012 - Aceito para publicação: 01/10/2013

\begin{abstract}
Resumo
Com o objetivo de avaliar os requerimentos nutricionais e os efeitos da omissão de nutrientes no desenvolvimento de mudas de barbatimão (Stryphnodendron adstringens (Mart.) Coville), conduziuse um experimento com o uso da técnica de elemento faltante. Foram empregados 12 tratamentos, em um delineamento de blocos inteiramente casualizados, com oito repetições. Adotaram-se os seguintes tratamentos: completo 1 (adubado com N, P, K, S, B, Cu, Zn e calagem como fonte de Ca e $\mathrm{Mg}$ e corrigindo acidez do solo), completo 2 (completo 1 - calagem, tendo $\mathrm{CaSO}_{4} \cdot 2 \mathrm{H}_{2} \mathrm{O}$ e $\mathrm{MgSO}_{4} \cdot 7 \mathrm{H}_{2} \mathrm{O}$ como fontes de Ca e Mg sem corrigir acidez do solo), C1 - N, C1 - P, C1 - K, C1 - S, C1 - B, C1 - Zn, $\mathrm{C} 1$ - calagem, C2 - Ca, C2 - Mg e testemunha. Foram medidos diâmetros e alturas das plantas, as quais foram separadas em parte aérea e sistema radicular. As mudas foram secas e pesadas, sendo determinados os teores de nutrientes na matéria seca da parte aérea. O fósforo e o boro foram os nutrientes mais limitantes, e a calagem afetou negativamente o crescimento. A sequência de exigência nutricional apresentada pelas mudas de barbatimão em relação ao tratamento completo, em ordem decrescente, foi: $\mathrm{P}>\mathrm{B}>\mathrm{Zn}>\mathrm{Mg}>\mathrm{K}>\mathrm{N}>\mathrm{S}>\mathrm{Ca}$.

Palavras-chave: Nutriente faltante; cerrado; nutrição florestal.
\end{abstract}

\begin{abstract}
Growth and mineral nutrition in seedlings of barbatimão subjected to nutrient deprivation. Aiming to evaluate nutritional requirements and effects of nutrient depravation in developing of seedlings of barbatimão (Stryphnodendron adstringens (Mart.) Coville), we conducted an experiment in a green house. We employed 12 treatments in a fully randomized block design with eight replications. We applied the following treatments: complete 1 (C1-fertilized with $\mathrm{N}, \mathrm{P}, \mathrm{K}, \mathrm{S}, \mathrm{B}, \mathrm{Cu}, \mathrm{Zn}$ and lime as $\mathrm{Ca}$ and $\mathrm{Mg}$ sources), complete 2 (complete 1 - lime with $\mathrm{CaSO}_{4} \cdot 2 \mathrm{H}_{2} \mathrm{O}$ and $\mathrm{MgSO}_{4} \cdot 7 \mathrm{H}_{2} \mathrm{O}$ as $\mathrm{Ca}$ and $\mathrm{Mg}$ sources), C1-N, C1-P, C1-K, C1-S, C1-B, C1-Zn, C1-lime, C2-Ca, C2-Mg, and control. We measured diameters and heights and separated them into shoot and root system. We dried the samples, weighed them and determined the nutrients levels in shoot dry matter. As result, we identified that phosphorus and boron were the most limiting factor to growth, as well as liming had a negative effect on growth; the sequence of nutritional requirements presented by barbatimão seedlings in relation to complete treatment was: $\mathrm{P}>\mathrm{B}>\mathrm{Zn}>\mathrm{Mg}>\mathrm{K}>\mathrm{N}>\mathrm{S}>\mathrm{Ca}$.
\end{abstract}

Keywords: Nutrient missing; savannah; forest nutrition.

\section{INTRODUÇÃO}

Stryphnodendron adstringens (Mart.) Coville, popularmente conhecida como barbatimão, é uma planta com 4 a 5 metros de altura, com tronco de 20 a $30 \mathrm{~cm}$ de diâmetro. Rica em taninos, na medicina popular a casca serve como anti-inflamatório e cicatrizante, sendo também indicada para diarreias, hemorragias, úlceras, uretrites e calvície. A madeira produz cerne vermelho e durável, de uso regional. A casca produz corante vermelho. As cinzas da madeira servem para fazer sabão. A espécie cresce no cerrado brasileiro, desde o Pará, na região Amazônica, até o Planalto Central, alcançando o Sudeste (Minas Gerais e São Paulo) (FELFILI et al., 1999; LORENZI, 2002; SILVA JÚNIOR, 2005). 
Em geral, as espécies florestais apresentam características distintas de comportamento, principalmente quanto às exigências nutricionais. $O$ conhecimento do status nutricional peculiar a cada espécie gera maior produtividade e economia e menores impactos ambientais nos plantios florestais.

Uma maneira rápida e econômica de se realizar esses estudos é através da utilização da técnica do nutriente faltante, que avalia qualitativamente um dado nutriente no solo. Essa técnica é simples e segura para a identificação de deficiências nutricionais. A mencionada técnica consiste em avaliar o desenvolvimento de uma espécie em casa de vegetação ou em campo, através de um tratamento completo (com todos os nutrientes necessários em doses adequadas) e uma série de tratamentos, nos quais é feita a omissão de um nutriente de cada vez (SANCHEZ; SALINAS, 1981). Para evitar que o resultado seja influenciado pela deficiência de outros nutrientes, adiciona-se fonte dos demais nutrientes, em quantidades não limitantes e nem tóxicas (BRAGA et al., 1995).

De acordo com Chaminade (1972), a técnica do nutriente faltante indica quais são os nutrientes que se apresentam deficientes, a importância relativa dessa deficiência e a velocidade de redução da fertilidade do solo. Para Malavolta (1980), ela apresenta uma referência semiquantitativa da necessidade de adubação. Vários trabalhos têm sido realizados utilizando a técnica com espécies florestais, como cedro australiano (Toona ciliata), cedro (Cedrela fissilis) e candeia (Eremanthus erythropappus) (MORETTI, et al., 2011; SOUZA et al., 2009; VENTURIN et al., 2005).

Tendo em vista a ausência de informações sobre as exigências nutricionais de barbatimão, esse trabalho teve como objetivo avaliar o status nutricional e os efeitos da omissão de nutrientes no desenvolvimento de mudas da espécie.

\section{MATERIAL E MÉTODOS}

O trabalho foi conduzido em casa de vegetação do Departamento de Ciências Florestais na Universidade Federal de Lavras (UFLA). Como substrato, foi utilizado um Latossolo Vermelho-Amarelo, de baixa fertilidade natural, coletado em uma área de cerrado no município de Itumirim, MG, a uma profundidade de 0,20 a $0,40 \mathrm{~m}$. Evitou-se coletar material pertencente à camada fértil do solo, para não mascarar o efeito dos fertilizantes usados.

O solo foi seco, peneirado e armazenado em sacos plásticos até serem adicionados os nutrientes utilizados na técnica do nutriente faltante. Foi realizada a incubação por um período de vinte dias. Ao final da incubação, o solo foi depositado em vasos com capacidade de $3,8 \mathrm{~kg}$ de solo. Os vasos constituíram as parcelas que ficaram localizadas sobre uma bancada, na casa de vegetação, com os fundos vedados para evitar perda de nutrientes.

Tabela 1. Identificação e caracterização dos tratamentos.

Table 1. Identification and characterization of treatments.

\begin{tabular}{lc}
\hline Tratamento & Caracterização \\
\hline Completo 1 (C1) & Aplicação de N, P, K, S, B, Cu, Zn + calcário \\
Completo 2 (C2) & Completo 1 - calcário + CaSO $4.2 \mathrm{H}_{2} \mathrm{O}$ e $\mathrm{MgSO}_{4} .7 \mathrm{H}_{2} \mathrm{O}$ \\
$\mathrm{C} 1-\mathrm{Cal}$ & Completo 1 - calcário \\
$\mathrm{C} 1-\mathrm{N}$ & Completo 1 - N \\
$\mathrm{C} 1-\mathrm{P}$ & Completo $1-\mathrm{P}$ \\
$\mathrm{C} 1-\mathrm{K}$ & Completo $1-\mathrm{K}$ \\
$\mathrm{C} 1-\mathrm{S}$ & Completo $1-\mathrm{S}$ \\
$\mathrm{C} 1-\mathrm{B}$ & Completo $1-\mathrm{B}$ \\
$\mathrm{C} 1-\mathrm{Zn}$ & Completo $1-\mathrm{Zn}$ \\
$\mathrm{C} 2-\mathrm{Ca}$ & Completo 2 - Ca \\
$\mathrm{C} 2-\mathrm{Mg}$ & Completo 2 - Mg \\
Test & Solo natural \\
\hline
\end{tabular}

Foram testados 12 tratamentos em um delineamento experimental de blocos inteiramente casualizados, com oito repetições, sendo um vaso por repetição e uma planta por vaso (Tabela 1). Para os tratamentos que receberam calcário, as doses foram baseadas no método de aumentar saturação por bases (V) a 70\%. O corretivo foi o calcário dolomítico calcinado, micropulverizado, com 36\% de CaO e 
$14 \%$ de $\mathrm{MgO}$ (PRNT igual a 100\%). No tratamento $\mathrm{C} 2$, o Ca e o $\mathrm{Mg}$ foram fornecidos, respectivamente, na forma de $\mathrm{CaSO}_{4} \cdot 2 \mathrm{H}_{2} \mathrm{O}$ e $\mathrm{MgSO}_{4} \cdot 7 \mathrm{H}_{2} \mathrm{O}$, tendo como finalidade somente o fornecimento de $\mathrm{Ca}$ e $\mathrm{Mg}$ sem alterar as demais características do solo.

As doses das fontes para o tratamento completo foram calculadas atendendo a adubação básica, de acordo com Raij (1991): $100 \mathrm{mg}$ de N, $300 \mathrm{mg}$ de P, $100 \mathrm{mg}$ de K, $200 \mathrm{mg}$ de Ca, $60 \mathrm{mg}$ de Mg, $40 \mathrm{mg}$ de $\mathrm{S}, 0,5 \mathrm{mg}$ de $\mathrm{B}, 1,5 \mathrm{mg}$ de $\mathrm{Cu}, 0,5 \mathrm{mg}$ de $\mathrm{Zn}$. Foram utilizados como fontes os seguintes sais p.a.: $\mathrm{NH}_{4} \mathrm{NO}_{3}, \mathrm{H}_{3} \mathrm{PO}_{4}, \mathrm{KH}_{2} \mathrm{PO}_{4}, \mathrm{MgSO}_{4}, \mathrm{CaSO}_{4} .2 \mathrm{H}_{2} \mathrm{O}, \mathrm{Na}_{2} \mathrm{SO}_{4}, \mathrm{CuSO}_{4} .5 \mathrm{H}_{2} \mathrm{O}, \mathrm{H}_{3} \mathrm{BO}_{3}$ e $\mathrm{ZnCl}_{2}$.

Com a finalidade de comprovar o efeito da adubação, foi realizada a análise do solo natural e do tratamento completo (Tabela 2). As análises físicas do solo constaram de determinação da textura (método do densímetro) e da densidade de partículas (método do balão volumétrico), conforme preconizado por EMBRAPA (1997). Elas foram realizadas no Laboratório de Física do Solo da Universidade Federal de Lavras. As análises químicas foram feitas através dos seguintes métodos: $\mathrm{pH}$ $\left(\mathrm{H}_{2} \mathrm{O}\right.$ - Relação 1:2,5); matéria orgânica (método de Walkley e Black, 1934); P e K (HCl 0,05 molc L ${ }^{-1}+$ $\mathrm{H}_{2} \mathrm{SO}_{4}$ 0,025 molc L ${ }^{-1}$ ), segundo Vettori (1969); $\mathrm{Ca}, \mathrm{Mg}, \mathrm{Al} \mathrm{e} \mathrm{H}+\mathrm{Al}$ (extrator $\mathrm{KCl} 1$ molc L$^{-1}$ ); $\mathrm{Zn}, \mathrm{Cu}$, $\mathrm{Fe}$ e $\mathrm{Mn}\left(\mathrm{HCl} 0,05\right.$ molc L $\mathrm{L}^{-1}+\mathrm{H}_{2} \mathrm{SO}_{4} 0,25$ molc L ${ }^{-1}$ ), segundo Viets Junior e Lindsay (1973); $\mathrm{S}\left(\mathrm{Ca}\left(\mathrm{H}_{2} \mathrm{PO}_{4}\right) \cdot \mathrm{H}_{2}+500 \mathrm{ppm} \mathrm{P}\right)$, conforme Tedesco et al. (1985); B (água quente) segundo descrição de Jackson (1970). Essas análises foram realizadas no Laboratório de Fertilidade do Solo da Universidade Federal de Lavras.

Tabela 2. Componentes físicos e químicos do solo natural e após adubação com macro e micronutrientes para barbatimão.

Table 2. Chemical and physical components of the natural soil and after fertilization with macro and micronutrients to barbatimão.

\begin{tabular}{lcc}
\hline Parâmetros & Solo natural & $\begin{array}{c}\text { Solo após adubação } \\
\text { completa }\end{array}$ \\
\hline $\mathrm{pH}\left(\mathrm{H}_{2} \mathrm{O}\right)$ & 5,4 & 5,6 \\
$\mathrm{P}\left(\mathrm{mg} / \mathrm{dm}^{3}\right)$ & 0,6 & 16,9 \\
$\mathrm{~K}\left(\mathrm{mg} / \mathrm{dm}^{3}\right)$ & 25 & 268 \\
$\mathrm{Ca}^{2+}\left(\mathrm{cmol}_{\mathrm{c}} / \mathrm{dm}^{3}\right)$ & 0,4 & 0,9 \\
$\mathrm{Mg}^{2+}\left(\mathrm{mg} / \mathrm{dm}^{3}\right)$ & 0,2 & 0,3 \\
$\mathrm{Al}^{3+}\left(\mathrm{cmol}_{\mathrm{c}} / \mathrm{dm}^{3}\right)$ & 0 & 0 \\
$\mathrm{H}+\mathrm{Al}\left(\mathrm{cmol}_{\mathrm{c}} / \mathrm{dm}^{3}\right)$ & 1,9 & 1,7 \\
$\mathrm{SB}\left(\mathrm{cmol}_{\mathrm{c}} / \mathrm{dm}^{3}\right)$ & 0,7 & 1,9 \\
$\mathrm{t}\left(\mathrm{cmol}_{\mathrm{c}} / \mathrm{dm}^{3}\right)$ & 0,7 & 1,9 \\
$\mathrm{~T}\left(\mathrm{cmol}_{\mathrm{c}} / \mathrm{dm}^{3}\right)$ & 2,6 & 3,6 \\
$\mathrm{~V}(\%)$ & 25,8 & 52,9 \\
$\mathrm{~m}(\%)$ & 0 & 0 \\
$\mathrm{MO}(\mathrm{dag} / \mathrm{kg})$ & 0,5 & 0,4 \\
$\mathrm{P}-\mathrm{rem}(\mathrm{mg} / \mathrm{L})$ & 9,4 & 19,9 \\
$\mathrm{Zn}\left(\mathrm{mg} / \mathrm{dm}^{3}\right)$ & 0,3 & 3,1 \\
$\mathrm{Fe}\left(\mathrm{mg} / \mathrm{dm}^{3}\right)$ & 12,6 & 11,8 \\
$\mathrm{Mn}\left(\mathrm{mg} / \mathrm{dm}^{3}\right)$ & 1,8 & 1 \\
$\mathrm{Cu}\left(\mathrm{mg} / \mathrm{dm}^{3}\right)$ & 0,4 & 2,2 \\
$\mathrm{~B}\left(\mathrm{mg} / \mathrm{dm}^{3}\right)$ & 0,3 & 0,3 \\
$\mathrm{~S}\left(\mathrm{mg} / \mathrm{dm}^{3}\right)$ & 20,7 & 26,9 \\
$\mathrm{Areia}(\mathrm{dag} / \mathrm{kg})$ & 68 & 66 \\
$\mathrm{Silte}(\mathrm{dag} / \mathrm{kg})$ & 7 & 7 \\
$\mathrm{Argila}(\mathrm{dag} / \mathrm{kg})$ & 25 & 27 \\
\hline
\end{tabular}

As sementes de Stryphnodendron adstringens foram coletadas no município de Lavras e, por possuírem dormência tegumentar, passaram por uma etapa de escarificação, visando aumentar o percentual de germinação. Para tal, foi utilizado ácido sulfúrico concentrado por cerca de 5 minutos, seguido de uma lavagem em água corrente por 1 hora e posterior imersão em água por 24 horas (DAVIDE; SILVA, 2008). Na etapa seguinte, as sementes, após a quebra da dormência, foram pré- 
germinadas em germinadores do laboratório de sementes florestais do DCF/UFLA, e assim que ocorreu a protrusão da radícula as sementes foram transferidas para os vasos com os tratamentos.

A umidade do solo foi mantida em torno de $60 \%$ do volume total de poros (VTP), conforme proposto por Freire et al. (1979), e aferida diariamente, através de pesagem, completando-se o peso com água deionizada.

O ensaio teve duração de oito meses. Ao término, os blocos foram desmontados e as raízes separadas do solo por lavagem em água corrente, obtendo-se as mudas inteiras e individualizadas, sendo realizada a medição do diâmetro do colo $(\mathrm{D})$ e da altura $(\mathrm{H})$, medida do nível do solo ao meristema da gema apical da planta.

Em seguida foi realizada a separação das plantas em parte aérea e sistema radicular, sendo o material levado para secagem em estufa de circulação forçada a $70{ }^{\circ} \mathrm{C}$, até atingir peso constante. Os materiais secos foram pesados, obtendo-se valores de matéria seca da parte aérea, matéria seca do sistema radicular e matéria seca total.

Com os valores de altura, diâmetro e com os pesos secos de sistema radicular, parte aérea e total, foi possível calcular o índice de qualidade de mudas de Dickson (IQD) (DICKSON et al., 1960). O IQD foi calculado pela fórmula:

$$
I Q D=\frac{M S T}{\left(\frac{H}{D}\right)+\left(\frac{M S P A}{M S R}\right)}
$$

Em que: IQD = índice de qualidade mudas de Dickson;

MST = matéria seca total;

$\mathrm{H}=$ altura da planta até a gema apical;

$\mathrm{D}=$ diâmetro do colo;

MSPA = matéria seca da parte aérea;

MSR = matéria seca do sistema radicular.

O material seco da parte aérea (caule, folhas e pecíolos) foi triturado em moinho tipo Willey e em seguida foram efetuadas as análises químicas para determinação dos teores totais de macro e micronutrientes, segundo Sarruge e Haag (1974). As análises foram feitas no Laboratório de Nutrição Mineral de Plantas, do Departamento de Ciências do Solo da UFLA.

Os dados foram submetidos aos testes de Shapiro-Wilk e Bartlett, a fim de verificar os pressupostos de normalidade e homogeneidade de variância, respectivamente. Uma vez atendidos esses pressupostos, aplicou-se o teste $\mathrm{F}$, através da análise de variância, e em seguida o teste de Scott-Knott a $5 \%$ de probabilidade, para comparar as médias entre os tratamentos, conforme Gomes (1985). Para tal, foi utilizado o programa Sisvar 4.6. Os gráficos e tabelas foram gerados pelo programa Microsoft Excel 2003.

\section{RESULTADOS E DISCUSSÃO}

\section{Crescimento das plantas}

Os resultados de altura, diâmetro do colo e produção de matéria seca, relação raiz/parte aérea e índice de qualidade de mudas de Dickson (IQD) de mudas de barbatimão nos diferentes tratamentos são apresentados na tabela 3 .

Para a variável diâmetro, os tratamentos com omissão de $\mathrm{P}$ e o testemunha foram os que apresentaram menor crescimento (Tabela 3), evidenciando que o P é um nutriente importante para o crescimento das mudas de barbatimão. O P é o nutriente mineral que se destaca por desempenhar papelchave em todos os metabólitos relacionados com a aquisição, estocagem e utilização de energia (açúcares fosfatados, adenosinas fosfatadas e em nucleotídeos e ácidos nucleicos), sendo portanto um elemento essencial para o genoma e para a aquisição de energia (EPSTEIN; BLOOM, 2004).

Os tratamentos com ausência de $\mathrm{Ca}$ apresentaram diâmetros superiores aos tratamentos completos (Figura 1). Isso pode indicar que a espécie está adaptada a solos ácidos e pobres em Ca. Sorreano (2006) encontrou resultados semelhantes para Cecropia pachystachya (embaúba), Lochocarpus muehlbergianus (embira-de-sapo), Cariniana legalis (jequitibá-rosa), Enterolobium contortisolliquum 
(orelha-de-nego) e Agiphila sellowiana (tamanqueiro). Note-se que, no geral, as espécies citadas são pioneiras adaptadas a solos pobres.

Os tratamentos sob omissão de $\mathrm{Ca}, \mathrm{S}$ e $\mathrm{K}$ tiveram valores semelhantes ao completo para o crescimento em altura. Souza et al. (2006) obtiveram resultados semelhantes em relação à ausência de Ca para outra espécie encontrada em solos de cerrado (Tabebuia impetiginosa), o que pode ser um indicador da adaptação da espécie a baixos teores de cálcio.

Tabela 3. Altura (H), diâmetro do colo (D), matéria seca da parte aérea (MSPA), matéria seca de raiz (MSR), matéria seca total (MST), relação raiz/parte aérea (R/PA) e índice de qualidade de Dickson para mudas de barbatimão nos diferentes tratamentos, aos 240 dias após transplantio.

Table 3. Height (H), diameter (D), dry weight of aerial plant (MSPA), dry root biomass (MSR), total dry biomass (MST), relation root/aerial (R/PA) and quality index of Dickson (IQD) to seedlings of barbatimão in different treatments, 240 days after transplanting.

\begin{tabular}{lccccccc}
\hline Tratamentos & Altura $(\mathbf{c m})$ & Diâmetro $(\mathbf{m m})$ & MSPA $(\mathbf{g})$ & MSR $(\mathbf{g})$ & MST $(\mathbf{g})$ & R/PA & IQD \\
\hline $\mathrm{C} 1$ & $16,50 \mathrm{~b}$ & $1,95 \mathrm{c}$ & $1,325 \mathrm{c}$ & $0,763 \mathrm{c}$ & $2,088 \mathrm{~d}$ & $0,61 \mathrm{~b}$ & $0,204 \mathrm{~d}$ \\
$\mathrm{C} 1-\mathrm{N}$ & $16,84 \mathrm{~b}$ & $2,21 \mathrm{c}$ & $2,311 \mathrm{c}$ & $3,562 \mathrm{~b}$ & $5,873 \mathrm{c}$ & $2,12 \mathrm{~b}$ & $0,583 \mathrm{c}$ \\
$\mathrm{C} 1-\mathrm{P}$ & $6,38 \mathrm{c}$ & $1,21 \mathrm{~d}$ & $0,065 \mathrm{c}$ & $0,264 \mathrm{c}$ & $0,329 \mathrm{~d}$ & $4,53 \mathrm{a}$ & $0,061 \mathrm{~d}$ \\
$\mathrm{C} 1-\mathrm{K}$ & $26,31 \mathrm{a}$ & $3,47 \mathrm{~b}$ & $3,477 \mathrm{~b}$ & $1,736 \mathrm{c}$ & $5,213 \mathrm{c}$ & $0,50 \mathrm{~b}$ & $0,553 \mathrm{c}$ \\
$\mathrm{C} 1-\mathrm{cal}$ & $21,16 \mathrm{a}$ & $3,96 \mathrm{a}$ & $5,745 \mathrm{a}$ & $5,350 \mathrm{a}$ & $11,095 \mathrm{a}$ & $1,02 \mathrm{~b}$ & $1,715 \mathrm{a}$ \\
$\mathrm{C} 1-\mathrm{S}$ & $23,88 \mathrm{a}$ & $2,67 \mathrm{c}$ & $3,033 \mathrm{~b}$ & $3,754 \mathrm{~b}$ & $6,786 \mathrm{~b}$ & $1,34 \mathrm{~b}$ & $0,699 \mathrm{c}$ \\
$\mathrm{C} 1-\mathrm{B}$ & $10,50 \mathrm{c}$ & $1,91 \mathrm{c}$ & $0,709 \mathrm{c}$ & $0,709 \mathrm{c}$ & $1,418 \mathrm{~d}$ & $1,52 \mathrm{~b}$ & $0,208 \mathrm{~d}$ \\
$\mathrm{C} 1-\mathrm{Zn}$ & $14,64 \mathrm{~b}$ & $2,24 \mathrm{c}$ & $1,467 \mathrm{c}$ & $1,416 \mathrm{c}$ & $2,883 \mathrm{~d}$ & $1,20 \mathrm{~b}$ & $0,408 \mathrm{c}$ \\
$\mathrm{C} 2$ & $24,06 \mathrm{a}$ & $2,97 \mathrm{~b}$ & $3,844 \mathrm{~b}$ & $5,449 \mathrm{a}$ & $9,293 \mathrm{a}$ & $1,40 \mathrm{~b}$ & $1,075 \mathrm{~b}$ \\
$\mathrm{C} 2-\mathrm{Ca}$ & $27,07 \mathrm{a}$ & $4,12 \mathrm{a}$ & $5,664 \mathrm{a}$ & $6,054 \mathrm{a}$ & $11,718 \mathrm{a}$ & $1,25 \mathrm{~b}$ & $1,569 \mathrm{a}$ \\
$\mathrm{C} 2-\mathrm{Mg}$ & $13,25 \mathrm{~b}$ & $2,08 \mathrm{c}$ & $1,575 \mathrm{c}$ & $2,115 \mathrm{c}$ & $3,690 \mathrm{c}$ & $1,52 \mathrm{~b}$ & $0,497 \mathrm{c}$ \\
Test & $7,56 \mathrm{c}$ & $1,20 \mathrm{~d}$ & $0,244 \mathrm{c}$ & $1,102 \mathrm{c}$ & $1,345 \mathrm{~d}$ & $5,28 \mathrm{a}$ & $0,211 \mathrm{~d}$ \\
\hline
\end{tabular}

Letras distintas na coluna diferem entre si pelo teste de Scott-Knott a 5\% de probabilidade.

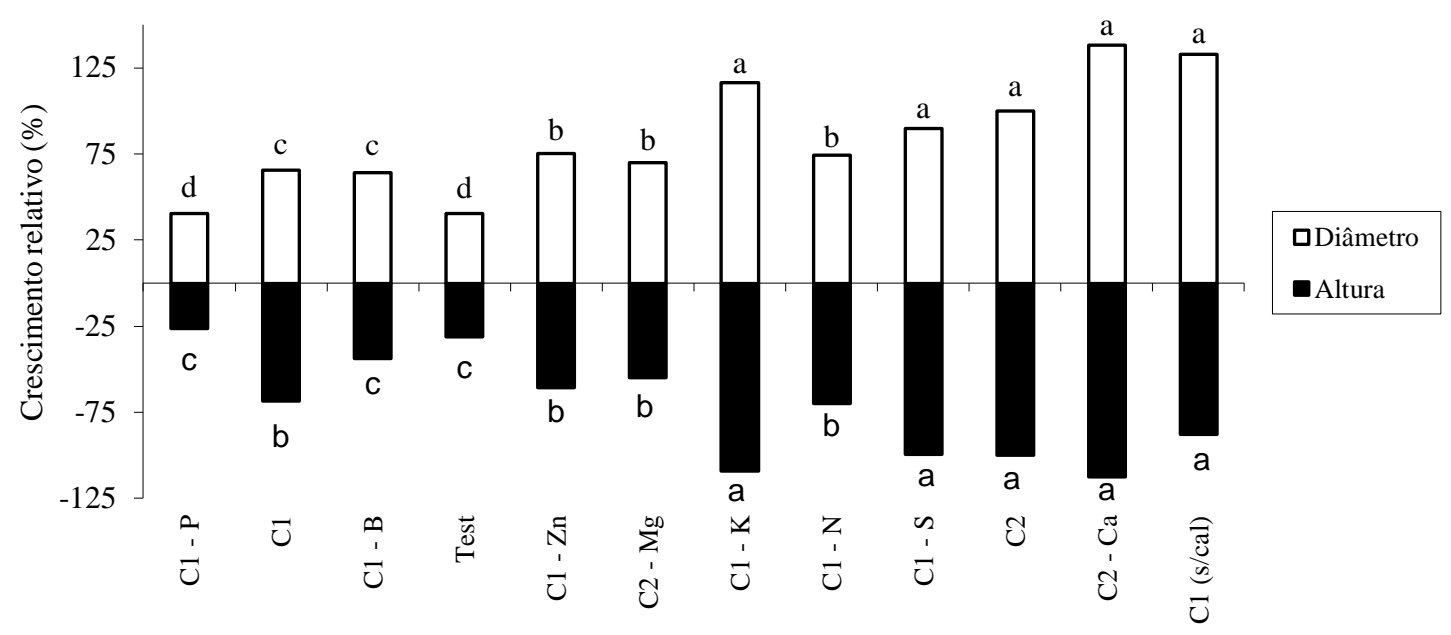

Letras distintas nas barras diferem entre si pelo teste de Scott-Knott a 5\% de probabilidade.

Figura 1. Crescimento relativo (\%) em diâmetro e altura para mudas de barbatimão.

Figure 1. Relative growth (\%) in diameter and height for barbatimão seedlings.

Os tratamentos com ausência de Ca apresentaram maior crescimento da matéria seca da parte aérea e raiz, sendo o crescimento radicular o mais prejudicado (Figura 2). Os tratamentos com ausência de $\mathrm{S}$ e K obtiveram resultados semelhantes ao tratamento completo 2. Venturin et al. (2005) obtiveram 
resultados semelhantes para Eremanthus erythropapus (candeia) e comentam que esse fato indica adaptação a tais ambientes de baixa fertilidade e solos ácidos.

Considerando a produção de matéria seca da parte aérea, diâmetro e altura, a sequência de exigência nutricional apresentada pelas mudas de barbatimão em relação ao tratamento completo, em ordem decrescente, foi: $\mathrm{P}>\mathrm{B}>\mathrm{Zn}>\mathrm{Mg}>\mathrm{K}>\mathrm{N}>\mathrm{S}>\mathrm{Ca}$.

A relação raiz/parte aérea para o barbatimão mostra que a espécie investe muito em sistema radicular e que, na ausência do $\mathrm{P}$, o que é comum nos ambientes naturais da espécie, ela aumenta a quantidade de raízes, buscando uma maior absorção dos nutrientes no solo. Em ambientes que apresentam baixa fertilidade, a relação R/PA é maior. A deficiência por fósforo gera um aumento da produção de raízes, que tendem a ficar mais longas e finas, aumentando assim a possibilidade de absorver fósforo, que é pouco móvel no solo. Isso pode ser explicado em parte pelo aumento das concentrações do ácido abscísico e diminuição da citocinina (MARSCHENER, 2011).

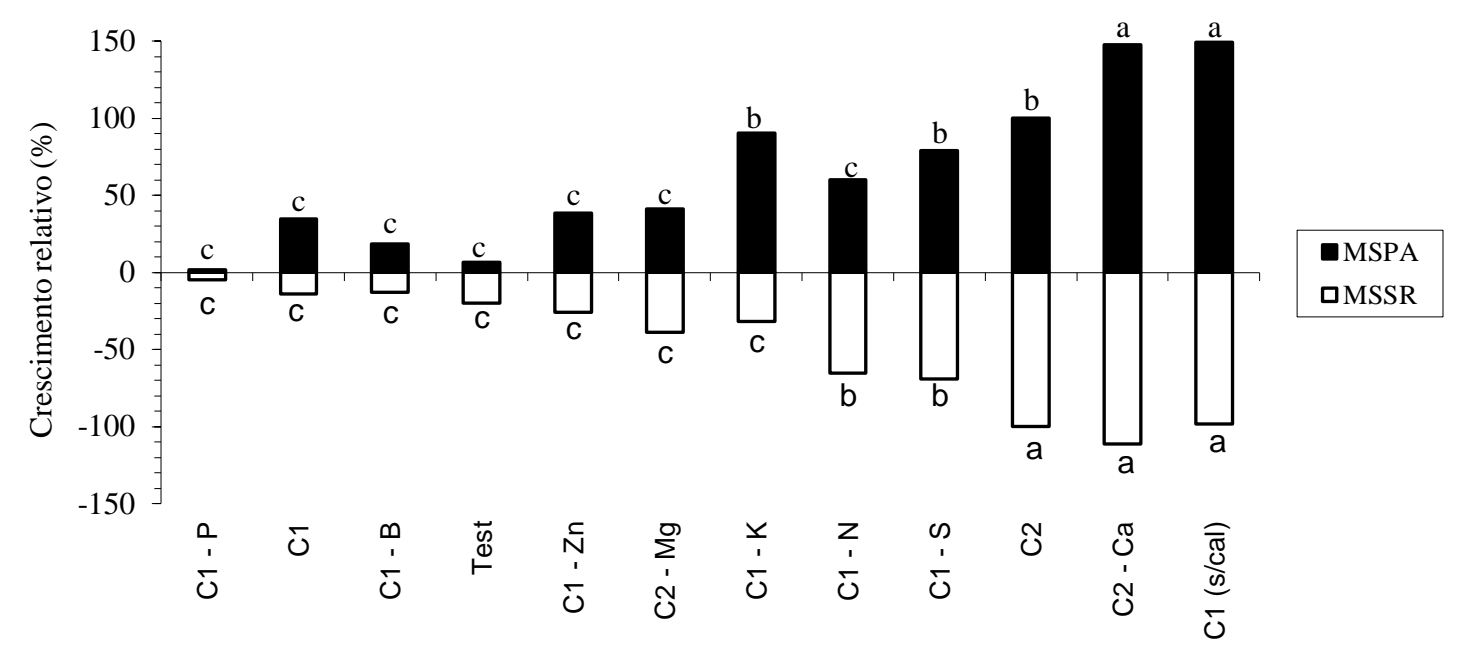

Letras distintas nas barras diferem entre si pelo teste de Scott-Knott a 5\% de probabilidade.

Figura 2. Crescimento relativo (\%) em matéria seca da parte aérea e sistema radicular para mudas de barbatimão.

Figure 2. Relative growth (\%) in dry biomass of shoots and roots for barbatimão seedlings.

Segundo Fonseca et al. (2002), o índice de qualidade de Dickson (IQD) é um bom indicador da qualidade das mudas, pois, no seu cálculo, são consideradas a robustez e o equilíbrio da distribuição da biomassa na muda, ponderando os resultados de vários parâmetros importantes empregados para avaliação da qualidade. Os resultados mostram que os tratamentos com omissão de Ca foram os que apresentaram os maiores valores de IQD, apresentando, portanto, mudas mais robustas e de melhor qualidade. Por outro lado, o menor IQD foi obtido nos tratamentos com ausência de P e B.

Como observado nas figuras 1 e 2, a ausência de calagem apresentou maiores valores de crescimento relativo, podendo-se assim dizer que a espécie não é exigente em calagem e que possui capacidade de absorver cálcio de solos mesmo com baixas concentrações, sendo que essas concentrações baixas nos seus tecidos (Tabela 4) são suficientes para as funções desempenhadas pelo cálcio na planta.

Furtini Neto et al. (1999) afirmam que a resposta em crescimento em função da correção da acidez do solo é sensivelmente maior para as espécies florestais de crescimento mais rápido, independentemente do seu grupo sucessional.

Considerando os resultados obtidos, pode-se afirmar que o barbatimão é uma espécie de crescimento lento e adaptada a ambientes ácidos, com baixos teores de $\mathrm{Ca}$ e, por conseguinte, não necessitando de calagem para formação de suas mudas. 


\section{Nutrição mineral do barbatimão}

Os teores dos nutrientes na matéria seca da parte aérea (MSPA) das mudas de barbatimão apresentaram-se baixos, de acordo com os tratamentos sob omissão de cada nutriente (Tabela 4).

Os tratamentos com omissão de $\mathrm{N}$ e $\mathrm{Mg}$ apresentaram as menores médias de nitrogênio, diferenciando-se estatisticamente dos demais. $\mathrm{O}$ tratamento com omissão de $\mathrm{K}$ apresentou teores de $\mathrm{N}$ estatisticamente semelhantes ao tratamento completo. Resultados semelhantes foram apresentados por Souza et al. (2006) para Eremanthus eritropapus (candeia). Esses autores encontraram um teor de 2,47 g.kg ${ }^{-1}$, semelhante ao resultado apresentado para barbatimão.

Os menores teores de fósforo foram encontrados nos tratamentos com omissão do mesmo e com omissão de N, B, Zn e Mg. Na nutrição mineral de plantas, o fósforo destaca-se por sua importância no metabolismo das plantas, estando presente em diversas atividades bioquímicas, fazendo parte do DNA, RNA e ATP, entre outros (EPSTEIN; BLOOM, 2004), sendo que sua falta afeta o desenvolvimento das plantas. O tratamento completo e com ausência de $\mathrm{K}$ foram os que apresentaram maiores teores de $\mathrm{P}$. Resultados semelhantes foram encontrados por Vieira et al. (2011) para Amburana acreana (cerejeira) e por Marques et al. (2004) para mudas de Schizolobium amazonicum (paricá).

Tabela 4. Teor de nutrientes na matéria seca da parte aérea (MSPA) de mudas de barbatimão submetidas a tratamentos com e sem omissão de nutrientes aos 240 dias após transplantio.

Table 4. Nutrient content in dry matter of aerial plant (MSPA) of barbatimão seedlings subjected to treatments with and without omission of nutrients 240 days after transplanting.

\begin{tabular}{lcccccccc}
\hline Tratamentos & $\mathbf{N}$ & $\mathbf{P}$ & $\mathbf{K}$ & $\mathbf{C a}$ & $\mathbf{M g}$ & $\mathbf{S}$ & $\begin{array}{c}\mathbf{B} \\
\mathbf{( g / k g})\end{array}$ & $\begin{array}{c}\mathbf{Z n} \\
\text { C1 }\end{array}$ \\
\hline & $20,2 \mathrm{a}$ & $0,78 \mathrm{a}$ & $5,4 \mathrm{~d}$ & $7,71 \mathrm{c}$ & $1,05 \mathrm{c}$ & $2,89 \mathrm{c}$ & $25,84 \mathrm{c}$ & $43 \mathrm{~b}$ \\
$\mathrm{C} 1-\mathrm{N}$ & $11,4 \mathrm{~d}$ & $0,47 \mathrm{c}$ & $3,8 \mathrm{e}$ & $10,88 \mathrm{~b}$ & $1,16 \mathrm{c}$ & $3,69 \mathrm{c}$ & $38,50 \mathrm{~b}$ & $22 \mathrm{c}$ \\
$\mathrm{C} 1-\mathrm{P}$ & $18,9 \mathrm{~b}$ & $0,40 \mathrm{c}$ & $13,4 \mathrm{a}$ & $7,62 \mathrm{c}$ & $2,29 \mathrm{a}$ & $2,58 \mathrm{c}$ & $52,34 \mathrm{a}$ & $62 \mathrm{a}$ \\
$\mathrm{C} 1-\mathrm{K}$ & $22,0 \mathrm{a}$ & $0,68 \mathrm{a}$ & $1,8 \mathrm{f}$ & $5,53 \mathrm{~d}$ & $1,42 \mathrm{c}$ & $2,75 \mathrm{c}$ & $28,85 \mathrm{c}$ & $49 \mathrm{~b}$ \\
$\mathrm{C} 1-\mathrm{Cal}$ & $17,8 \mathrm{~b}$ & $0,56 \mathrm{~b}$ & $9,2 \mathrm{~b}$ & $2,73 \mathrm{e}$ & $0,70 \mathrm{~d}$ & $2,93 \mathrm{c}$ & $28,47 \mathrm{c}$ & $40 \mathrm{~b}$ \\
$\mathrm{C} 1-\mathrm{S}$ & $14,5 \mathrm{c}$ & $0,56 \mathrm{~b}$ & $6,0 \mathrm{~d}$ & $8,64 \mathrm{c}$ & $1,75 \mathrm{~b}$ & $2,82 \mathrm{c}$ & $41,85 \mathrm{~b}$ & $28 \mathrm{c}$ \\
$\mathrm{C} 1-\mathrm{B}$ & $17,9 \mathrm{~b}$ & $0,47 \mathrm{c}$ & $8,7 \mathrm{~b}$ & $6,51 \mathrm{~d}$ & $1,21 \mathrm{c}$ & $3,63 \mathrm{~b}$ & $21,47 \mathrm{c}$ & $59 \mathrm{a}$ \\
$\mathrm{C} 1-\mathrm{Zn}$ & $17,5 \mathrm{~b}$ & $0,42 \mathrm{c}$ & $7,2 \mathrm{c}$ & $7,00 \mathrm{~d}$ & $1,27 \mathrm{c}$ & $3,41 \mathrm{~b}$ & $37,55 \mathrm{~b}$ & $29 \mathrm{c}$ \\
$\mathrm{C} 2$ & $15,0 \mathrm{c}$ & $0,66 \mathrm{a}$ & $4,4 \mathrm{e}$ & $8,21 \mathrm{c}$ & $0,97 \mathrm{~d}$ & $2,77 \mathrm{c}$ & $50,41 \mathrm{a}$ & $28 \mathrm{c}$ \\
$\mathrm{C} 2-\mathrm{Mg}$ & $12,8 \mathrm{~d}$ & $0,42 \mathrm{c}$ & $9,0 \mathrm{~b}$ & $14,77 \mathrm{a}$ & $1,32 \mathrm{c}$ & $5,74 \mathrm{a}$ & $50,68 \mathrm{a}$ & $34 \mathrm{c}$ \\
$\mathrm{C} 2-\mathrm{Ca}$ & $19,1 \mathrm{~b}$ & $0,59 \mathrm{~b}$ & $6,2 \mathrm{~d}$ & $2,21 \mathrm{e}$ & $0,62 \mathrm{~d}$ & $2,39 \mathrm{c}$ & $25,58 \mathrm{c}$ & $48 \mathrm{~b}$ \\
Test & $16,5 \mathrm{~b}$ & $0,43 \mathrm{c}$ & $1,15 \mathrm{f}$ & $6,45 \mathrm{~d}$ & $0,83 \mathrm{~d}$ & $1,16 \mathrm{~d}$ & $30,30 \mathrm{c}$ & $22 \mathrm{c}$ \\
\hline
\end{tabular}

Letras distintas na coluna diferem entre si pelo teste de Scott-Knott a 5\% de probabilidade.

Os teores de $\mathrm{K}$ mais elevados foram encontrados no tratamento com ausência de $\mathrm{P}$, seguido pelos tratamentos com ausência de $\mathrm{Mg}$, calagem e $\mathrm{B}$. O alto teor de $\mathrm{K}$ no tratamento com ausência de fósforo possivelmente se deve a um efeito de concentração devido ao pouco crescimento da muda. Os elevados teores de $\mathrm{K}$ encontrados nas ausências de calagem $(\mathrm{Ca})$ e $\mathrm{Mg}$ estão relacionados à ausência do mecanismo de inibição competitiva entre esses nutrientes (MALAVOLTA, 1989). Resultados semelhantes foram obtidos por Sarcinelli et al. (2004) em mudas de Acácia holoserice e por Venturin et al. (1996) em Copaifera langsdorffii, em que os tratamentos com ausência de Ca e Mg apresentaram teores elevados de $\mathrm{K}$ quando comparados ao tratamento completo.

Os menores teores de $\mathrm{Ca}$ foram encontrados nos tratamentos com omissão desse nutriente, porém vale ressaltar que os tratamentos com ausência de $\mathrm{Ca}$ apresentaram maior crescimento, mostrando assim que essa é uma concentração adequada, pois não foi prejudicial às mudas, possivelmente devido à adaptação da planta a ambientes com pouco cálcio disponível no solo. Já os maiores teores foram encontrados nos tratamentos com omissão de $\mathrm{Mg}$ e $\mathrm{N}$. Esse resultado se dá possivelmente pela redução da competição entre Mg e Ca (MALAVOLTA, 1989). Resultados semelhantes foram encontrados por Mendonça et al. (1999) em mudas de Myracrodruon urundeuva e por Sarcinelli et al. (2004) para mudas de Acacia holosericea, em que os tratamentos na ausência de $\mathrm{Mg}$ apresentaram maiores teores de Ca. 
O maior teor de $\mathrm{Mg}$ foi encontrado no tratamento com ausência de P. Vichiato (2005), estudando a nutrição do mamoeiro, também verificou que os teores de $\mathrm{Mg}$ foram influenciados por interações $\mathrm{P}-\mathrm{Mg}$, sendo que houve decréscimos nos teores de $\mathrm{Mg}$ com o aumento das doses de $\mathrm{P}$.

O tratamento com omissão de $\mathrm{Mg}$, seguido pelo com omissão de B e $\mathrm{Zn}$, apresentou maiores teores de $\mathrm{S}$ na matéria seca da parte aérea. Esses resultados possivelmente estão relacionados com a concentração do $\mathrm{S}$, devido à baixa produção de matéria seca da parte aérea nos tratamentos com omissão de Mg e Zn. Resultados semelhantes foram encontrados por Venturin et al. (1996) em Copaifera langsdorffii e por Sorreano (2006) para mudas de Guazuma ulmifolia (mutambo).

Os maiores teores de B foram encontrados no tratamento completo e sob omissão de $\mathrm{P}$ e $\mathrm{Mg}$. Sob a omissão de $\mathrm{P}$, esses valores provavelmente ocorreram devido à concentração do teor de $\mathrm{B}$ nas mudas de barbatimão pela pouca produção de matéria seca da parte aérea.

Os tratamentos com omissão de P e B foram os tratamentos que apresentaram maiores teores de $\mathrm{Zn}$ na matéria seca da parte aérea. $\mathrm{O}$ alto teor de $\mathrm{Zn}$ no tratamento com ausência de $\mathrm{P}$ ocorre possivelmente pela concentração dos nutrientes na matéria seca da parte aérea, que apresentou crescimento menor que nos outros tratamentos. $\mathrm{O}$ elevado teor de $\mathrm{Zn}$ na ausência de $\mathrm{B}$ se dá devido à diminuição da inibição não competitiva entre o íon $\mathrm{Zn}^{2+}$ e o $\mathrm{H}_{2} \mathrm{BO}_{3}$ (MALAVOLTA, 1989), sendo que, com a redução do teor de $\mathrm{Zn}$, o B foi mais absorvido pelas plantas de barbatimão.

\section{CONCLUSÕES}

Os resultados obtidos nas condições do presente trabalho permitiram concluir que:

- O fósforo e o boro foram os nutrientes mais limitantes ao crescimento do barbatimão;

- A calagem afetou negativamente o crescimento, não sendo necessária na produção de mudas de barbatimão;

- A sequência de exigência nutricional apresentada pelas mudas de barbatimão em relação ao tratamento completo, considerando a produção de matéria seca da parte aérea, diâmetro e altura, em ordem decrescente, foi: $\mathrm{P}>\mathrm{B}>\mathrm{Zn}>\mathrm{Mg}>\mathrm{K}>\mathrm{N}>\mathrm{S}>\mathrm{Ca}$.

\section{REFERÊNCIAS}

BRAGA, F. A.; VALE, F. R.; VENTORIN, N.; AUBERT, E.; LOPES, G. A. Requerimentos nutricionais de quatro espécies florestais Revista Árvore, Viçosa, v. 19, n. 1, p. 18 - 32, 1995.

CHAMINADE, R. Recherches sur la fertilité et la fertilisation des sols en régions tropicales. Agronomie Tropicale, Montpellier, v. 27, p. 891 - 904, 1972.

DAVIDE, A. C.; SILVA, E. A. A. da. Produção de sementes e mudas de espécies florestais. Lavras: UFLA, 2008. $175 \mathrm{p}$.

DICKSON, A.; LEAF, A.; HOSNER, J. F. Quality appraisal of white spruce and white pine seedling stock in nurseries. Forestry Chronicle, v. 36, p. 10 - 13, 1960.

EMBRAPA. Manual de métodos de análise de solos. 2. ed. Rio de Janeiro: CNPS, 1997. 212 p.

EPSTEIN, E.; BLOOM, A. J. Nutrição mineral de plantas: princípios e perspectivas. 2. ed. Londrina: Planta, 2004. 403 p.

FELFILI, J. M.; SILVA JÚNIOR, M. C. da; DIAS, B. J.; REZENDE, A. V. Estudo fenológico de Stryphnodendron adstringens (Mart.) Coville no cerrado sensu stricto da Fazenda Água Limpa, no Distrito Federal, Brasil. Revista Brasileira de Botânica, Viçosa, v. 22, p. 83 - 90, 1999.

FONSECA, E. P.; VALÉRI, S. V.; MIGLIORANZA, E.; FONSECA, N. A. N.; COUTO, L. Padrão de qualidade de mudas de Trema micrantha (L.) Blume, produzidas sob diferentes períodos de sombreamento. Revista Árvore, Viçosa, v. 26, n. 4, p. 515 - 523, 2002.

FREIRE, J. C.; RIBEIRO, M. A. V.; BAHIA, V. G.; LOPES, A. S.; MOVAIS, R. F. Métodos de aplicação de adubos na formação de mudas de Eucalyptus grandis F. Hill ex Maiden. Silvicultura, São 
Paulo, v. 14, p. 385 - 386, 1979.

FURTINI NETO, A. E.; RESENDE, A. V.; VALE, F. R.; FAQUIN, V.; FERNANDES, L. A. Acidez do solo, crescimento e nutrição mineral de algumas espécies arbóreas, na fase de muda. Cerne, Lavras, v. 5, n. 2, p. $1-12,1999$.

GOMES, F. P. Curso de estatística experimental. 2.ed. São Paulo: Nobel, 1985. 466 p.

JACKSON, M. L. Análise química de suelos. Barcelona: Omega, 1970. 66 p.

LORENZI, H. Árvores brasileiras: manual de identificação e cultivo de plantas arbóreas nativas do Brasil. Nova Odessa: Plantarum, 2002. 368 p.

MALAVOLTA, E. Elementos de nutrição mineral de plantas. São Paulo: Ceres, 1980. 251 p.

. Avaliação do estado nutricional das plantas: princípios e aplicações. São Paulo: Associação Brasileira para Pesquisa da Potassa e do Fosfato, 1989. 201 p.

MARQUES, T. C. L. L. M.; CARVAlHO, J. G. de; LACERDA, M. P. C.; MOTA, P. E. F. da. Exigências nutricionais do paricá (Schizolobium amazonicum Herb.) na fase de muda. Revista Cerne, Lavras, v. 10, n. 2, p. 167 - 183, 2004.

MARSCHNER, H. Mineral nutrition of higher plants. 3. ed. London: Academic, 2011. 649 p.

MENDONÇA, A. V. R.; NOGUEIRA, F. D.; VENTURIN, N.; SOUZA, J. S. Exigências nutricionais de Myracrodruon urundeuva Fr. All. (aroeira do sertão). Revista Cerne, Lavras, v. 5, n. 2, p. 65 - 75, 1999.

MORETTI, B. S.; FURTINI NETO, A. E.; PINTO, S. I. C.; FURTINI, I. V.; MAGALHÃES, C. A. S. Crescimento e nutrição mineral de cedro australiano (Toona ciliata) sob omissão de nutrientes. Cerne, Lavras, v. 17, n. 4, p. 453 - 463, 2011.

RAIJ, B. V. Fertilidade do solo e adubação. São Paulo: Associação Brasileira para Pesquisa da Potassa e do Fosfato/Agronômica Ceres, 1991. 343 p.

SANCHEZ, P. A.; SALINAS, J. G. Low-input technology for managing oxisols and ultisols in tropical America. Advances in Agronomy, Madison, v. 34, p. 279 - 406, 1981.

SARCINELLI, T. S.; RIBEIRO JÚNIOR, E. S.; DIAS, L. E.; LYNCH, L. S. Sintomas de deficiência nutricional em mudas de Acacia holosericea em resposta à omissão de macronutrientes. Revista Árvore, Viçosa, v. 28, n. 2, p. 173 - 181, 2004.

SARRUGE, J. R.; HAAG, H. P. Análises químicas em plantas. Piracicaba: ESALQ/USP, 1974. 56 p.

SILVA JÚNIOR, M. C. da. 100 árvores do Cerrado: guia de campo. Brasília, DF: Ed. Rede de Sementes do Cerrado, 2005. 278 p.

SORREANO, M. C. M. Avaliação da exigência nutricional na fase inicial do crescimento de espécies florestais nativas. 296 p. Tese (Doutorado em Ecologia Aplicada) - Escola Superior de Agricultura "Luiz de Queiroz". Piracicaba, 2006.

SOUZA, P. A.; VENTURIN, N.; MACEDO, R. L. G. Adubação mineral do ipê-roxo (Tabebuia impetiginosa). Ciência Florestal, Santa Maria, v. 16, n. 3, p. 261 - 270, 2006.

SOUZA, P. A.; VENTURIN, N.; MACEDO, R. L. G.; VENTURIN, R. P.; TUCCI, C. A. F.; CARLOS, L. Nutritional assessment of cedar seedlings (cedrela fissilis Vell.) grown in a greenhouse. Cerne, Lavras, v. 15, n. 2, p. 236 - 243, 2009.

TEDESCO, M. J.; VOLKWEISS, S. J.; BOHNRN, H. Análise do solo, plantas e outros materiais. Porto Alegre: UFRGS, 1985. 188 p.

VENTURIN, N.; DUBOC, E.; VALE, F. R.; DAVIDE, A. C. Fertilização de plântulas de Copaifera langsdorffii Desf. (óleo copaíba). Revista Cerne, Lavras, v. 2, n. 2, p. 1 - 17, 1996.

VENTURIN, N.; SOUZA, P. A.; MACEDO, R. L. G.; NOGUEIRA, F. D. Adubação mineral da candeia 
(Eremanthus erythropappus (DC.) McLeish). Floresta, Curitiba, v. 35, n. 2, p. 211 - 219, 2005.

VETTORI, L. Métodos de análise de solo. Rio de Janeiro: Ministério da Agricultura, 1969. 34 p.

VICHIATO, M. Nutrição mineral e crescimento de mudas de mamoeiro em função de fósforo e magnésio. 2005. 90 p. Tese (Doutorado em Fitotecnia) - Universidade Federal de Lavras. Lavras, MG, 2005.

VIETS JUNIOR, F. G.; LINDSAY, W. L. Testing soils for zinc, Cooper, manganese and iron. In: WALSH, L. M.; BEATON, J. D. (Ed.). Soil testing and plant analylis. Madison: Soil Science Society of America, p. 329 - 488. 1973.

WALKLEY, A.; BLACK, I. A. An examination of the Degtjareff method for determining soil organic matter, and a proposed modification of the chromic acid titration method. Soil Science, Baltimore, v. 37, p. $29-38,1934$. 